\title{
A PROBLEM IN ARRANGEMENTS
}

BY M. H. MARTIN

A problem in dynamics, recently considered by the author, gave rise to the following question.

Let us consider the $n^{r}$ permutations of $n$ different symbols $e_{1}, e_{2}, \cdots, e_{n}$ taken $r$ at a time with repetitions allowed. Can a sequence of these symbols be constructed such that each of these $n^{r}$ permutations is found exactly once as a subsequence of $r$ consecutive symbols in this sequence?

Thus for $n=10$ and $r=5$ the question is equivalent to asking whether there exists a succession of digits such that every fiveplace number* is found exactly once among the consecutive digits of the succession. To consider a simpler example, in which the answer is readily given, let us take $n=3$ and $r=2$. Here the $n^{r}=3^{2}=9$ permutations are

$$
e_{1} e_{1}, e_{1} e_{2}, e_{1} e_{3}, e_{2} e_{1}, e_{2} e_{2}, e_{2} e_{3}, e_{3} e_{1}, e_{3} e_{2}, e_{3} e_{3},
$$

and a sequence possessing the desired properties is

$$
e_{1} e_{3} e_{3} e_{2} e_{3} e_{1} e_{2} e_{2} e_{1} e_{1} \text {. }
$$

We now return to the general case and show that the answer to the question in italics above is in the affirmative for any pair of positive integers $n$ and $r$ whatsoever. The proof proceeds by first exhibiting an algorithm, the application of which is then shown to lead to a sequence of the symbols $e_{1}, e_{2}, \cdots, e_{n}$ possessing the desired properties.

From now on a subsequence of $r$ consecutive symbols occurring in a sequence will always be designated as a section of $r$ symbols. Thus $e_{3} e_{2} e_{3} e_{1}$ is a section of 4 symbols in the sequence (1).

The algorithm in question is built up out of the following three rules.

I. Each of the first $r-1$ symbols is chosen equal to $e_{1}$.

II. The symbol $a_{m}$ to be added to the sequence

* By five-place numbers we mean, of course, those like 31342,41231 etc., including those, however, such as 00123,00005 , etc. 


$$
\begin{aligned}
& a_{1} a_{2} \cdots a_{r-1} a_{r} \cdots a_{m-r+1} \cdots a_{m-1}, \\
& a_{1}=a_{2}=\cdots=a_{r-1}=e_{1}, \quad m \geqq r,
\end{aligned}
$$

where the a's stand for the $e^{\prime}$ 's in a certain order, is the $e_{i}$ with the greatest subscript consistent with the requirement that the section $a_{m-r+1} \cdots a_{m-1} a_{m}$ duplicate no previously occurring section of $r$ symbols in (2).

III. Rule II is first applied for $m=r$ (in which case $a_{m}=a_{r}=e_{m}$ ) and is then applied repeatedly until a further application is impossible.

At this point the reader is urged to verify that the sequence (1) has been constructed by use of this algorithm.

Suppose that the application of Rule I followed by repeated applications of Rule II yields a certain sequence

$$
\begin{aligned}
& a_{1} a_{2} \cdots a_{r-1} a_{r} \cdots a_{p-r} a_{p-r+1} \cdots a_{p-1}, \\
& \quad a_{1}=a_{2}=\cdots=a_{r-1}=e_{1}, \quad p>r,
\end{aligned}
$$

of the $e$ 's. When may Rule II be applied once more to add another symbol, say $a_{p}$, to (3)? Before answering this question in the lemmas below we must point out that no two sections of $r$ symbols each in (3) are identical. This fact will be used in the proof of the following lemma.

LEMMA 1. If at least one of the symbols in the section $a_{p-r+1} \cdots$ $a_{p-1}$ of (3) differs from $e_{1}$, Rule II may be applied once more to add another symbol, say $a_{p}$, to (3).

In order to prove this lemma, let us count the number of times the section $a_{p-r+1} \cdots a_{p-1}$ of $r-1$ symbols in (3) has occurred prior to its occurrence in the right-hand end position. If it has never occurred prior to its occurrence in the right-hand end position, we are able to apply Rule II once more and add another symbol $a_{p}=e_{n}$ to (3). Clearly, if it has occurred $j,(j<n)$, times prior to its occurrence in the right-hand end position, Rule II may be applied once more to add $a_{p}=e_{n-j}$. In order to complete the proof of the lemma we show that under the hypothesis of the lemma (namely, that at least one of the symbols in the section $a_{p-r+1} \cdots a_{p-1}$ differs from $e_{1}$ ) the number of times the section $a_{p-r+1} \cdots a_{p-1}$ occurs prior to its occurrence in the right-hand end position is always less than $n$. For, suppose the contrary were true. The section $a_{p-r+1} \cdots a_{p-1}$ would 
then occur at least $n+1$ times in (3). Since at least one of the symbols contained in it differs from $e_{1}$, it cannot occupy the left-hand end position in (3) and hence is preceded by at least one symbol at each of its occurrences. On adjoining this preceding symbol to $a_{p-r+1} \cdots a_{p-1}$ at each of its occurrences we conclude that the sequence (3) contains the following $n+1$ sections of $r$ symbols each:

$$
b_{i} a_{p-r+1} \cdots a_{p-1}, \quad(i=1,2, \cdots, n+1),
$$

where $b_{i}$ stands for one of the $e$ 's. Since there are only $n$ of the $\boldsymbol{e}$ 's, at least two of the sections (4) must be identical and this is a contradiction to the property of the sequence (3) pointed out above when we introduced this lemma.

Obviously Rule II can only be applied a finite number of times. To answer the question as to when it can no longer be applied, we shall prove the following lemma.

LemMa 2. In order that Rule II can no longer be applied to add another symbol to (3) it is necessary and sufficient that each of the symbols in the section $a_{p-r} a_{p-r+1} \cdots a_{p-1}$ of (3) is equal to $e_{1}$.

To show the necessity of the condition suppose Rule II can not be applied to (3). Then, first of all, it follows from Lemma 1 that each of the symbols $a_{p-r+1}, \cdots, a_{p-1}$ is equal to $e_{1}$. Furthermore, the sequence (3) must contain the following $n$ sections of $r$ symbols each :

$$
e_{1} e_{1} \cdots \epsilon_{1} e_{n-j}, \quad(j=0,1, \cdots, n-1) ;
$$

otherwise Rule II could be applied to (3). The section $j=0$ in (5) occupies the left-hand end position in (3), the remaining sections $j=1,2, \cdots, n-1$ being distributed from left to right in (3) with increasing $j$. The section $j=n-1$ in (5) is composed exclusively of $e_{1}$ 's and occupies the right-hand end position in (3). For, suppose it did not. Suppress those symbols which would then lie to the right of it in (3). The sequence so obtained contains all of the sections in (5) and each of the last $r-1$ symbols in it is equal to $e_{1}$. Hence Rule II cannot be applied to add another symbol and this is a contradiction to the assumption that certain symbols lie to the right of the section $j=n-1$ of (5) in the sequence (3). Therefore our assumption that the sec- 
tion $j=n-1$ in (5) does not occupy the right-hand end position is false.

To show the sufficiency of the condition suppose that each of the symbols in the section $a_{p-r} a_{p-r+1} \cdots a_{p-1}$ of (3) is equal to $e_{1}$. From the nature of Rule II it follows that each of the sections in (5) occurs in (3). Since each of the last $r-1$ symbols in (3) is equal to $e_{1}$, Rule II cannot be applied to add another symbol.

Let us suppose that we have constructed a sequence

$$
\begin{array}{r}
a_{1} a_{2} \cdots a_{r-1} a_{r} \cdots a_{N-r+1} \cdots a_{N}, \quad a_{j}=a_{N-j}=a_{N}=e_{1}, \\
(j=1,2, \cdots, r-1),
\end{array}
$$

according to our algorithm. With respect to this sequence we shall prove the following theorem.

TheOREM. An arbitrarily given permutation of the symbols $e_{1}, e_{2}, \cdots, e_{n}$ taken $r$ at a time with repetitions allowed occurs exactly once as a section of $r$ symbols in the sequence (6).

That a given permutation can occur only once as a section of $r$ symbols in (6) is an immediate consequence of Rule II in the algorithm. That a given permutation actually occurs as a section of $r$ symbols in (6) is taken up next. First of all, since the last $r$ symbols in (6) are each equal to $e_{1}$, the sequence (6) contains each of the sections (5). (See the sufficiency proof of Lemma 2.) The section $j=0$ in (5) is the only one that occupies the left-hand position in (6). Since the section $j=n-1$ in (5) contains two sections of $r-1$ consecutive $e_{1}$ 's, a section of $r-1$ consecutive $e_{1}$ 's occurs $n$ times in (6) in addition to its occurrence there in the left-hand end position. Now no two sections of $r$ symbols can be identical in (6). Hence each of the sections

$$
e_{j} e_{1} e_{1} \cdots e_{1}, \quad(j=1,2, \cdots, n),
$$

of $r$ symbols each, occurs in (6). From the nature of Rule II in the algorithm and the presence of the sections (7) in (6) it follows that the sections

$$
e_{j} e_{1} e_{1} \cdots e_{1} e_{k}, \quad(j, k=1,2, \cdots, n),
$$

of $r$ symbols each, also occur in (6).

Let $b_{1} b_{2} b_{3} \cdots b_{r-1} b_{r}$ denote an arbitrary permutation of 
$e_{1}, e_{2}, \cdots, e_{n}$ with repetitions of a symbol allowed. We show that this permutation can be found as a section of $r$ symbols in (6). The proof of this fact has been given in the preceding paragraph if $b_{2}=b_{3}=\cdots=b_{r-1}=e_{1}$ and we rule this case out in the following. In order to show that $b_{1} b_{2} b_{3} \cdots b_{r-1} b_{r}$ occurs as a section of $r$ symbols in (6) it is clearly sufficient to show that $b_{1} b_{2} b_{3} \cdots b_{r-1} e_{1}$ occurs there as a section of $r$ symbols. Now suppose that $b_{1} b_{2} b_{3} \cdots b_{r-1} e_{1}$ does not occur as a section of $r$ symbols in (6). Since the section $b_{2} b_{3} \cdots b_{r-1} e_{1}$ contains, by hypothesis, a symbol different from $e_{1}$, it cannot occupy the lefthand end position in (6). Hence it can occur at most $n-1$ times in (6); otherwise the section $b_{1} b_{2} b_{3} \cdots b_{r-1} e_{1}$ would have to occur in (6) contrary to our hypothesis. Since the section $b_{2} b_{3} \cdots b_{r-1} e_{1}$ occurs at most $n-1$ times in (6), the section $b_{2} b_{3} \cdots b_{r-1} e_{1} e_{1}$ cannot occur in (6). Two possibilities are now open, namely:

(a) The section $b_{3} \cdots b_{r-1} e_{1} e_{1}$ contains no symbol different from $e_{1}$,

(b) The section $b_{3} \cdots b_{r-1} e_{1} e_{1}$ contains a symbol different from $e_{1}$.

If (a) presents itself, the conclusion that the section $b_{2} b_{3} \ldots$ $b_{r-1} e_{1} e_{1}$ cannot occur in (6) is a contradiction to the existence of the sections (7) in (6). Hence, in this case, our assumption that the section $b_{1} b_{2} b_{3} \cdots b_{r-1} e_{1}$ does not occur in (6) was false.

If (b) presents itself, a repetition of our earlier reasoning is used to show that the section $b_{3} b_{4} \cdots b_{r-1} e_{1} e_{1} e_{1}$ cannot occur in (6) and the discussion is repeated on the basis that this section cannot occur in (6), etc. Barring any earlier arrival to a contradiction of our results in (7) we must eventually reach the contradiction that the section $b_{r-1} e_{1} e_{1} \cdots e_{1}$ of $r$ symbols cannot occur in (6).

The question raised at the beginning of this paper is now disposed of and we close by pointing out an obvious extension to our results. Consider, as before, the $n^{r}$ permutations of $n$ different symbols $e_{1}, e_{2}, \cdots, e_{n}$ taken $r$ at a time with repetitions allowed. It is possible to construct a sequence of these symbols such that each of the $n$ permutations is found exactly $k$ times as a section of $r$ symbols in the sequence.

To show this let us first take $k=2$. Consider two sequences of the $e$ 's each identical with the sequence (6). From one of these 
sequences remove the first $r-1$ of the $e_{1}$ 's occurring in the lefthand end position and place this truncated sequence on the same horizontal line with the other and to its right. The resulting sequence of the $e$ 's obviously contains each of the $n^{r}$ permutations exactly twice.

The case of an arbitrary $k$ is then readily disposed of by successive applications of this process.

Trinity College

\section{CRITERIA FOR THE IRREDUCIBILITY OF POLYNOMIALS*}

\section{BY LOUIS WEISNER}

1. Introduction. If a polynomial with integral coefficients is reducible in the field of rational numbers, the task of decomposing it into the product of irreducible polynomials may be expected to involve a great deal of numerical work, commensurate with the degree and coefficients of the polynomial, such as is required by Kronecker's method. But when it is merely required to know whether or not the polynomial is reducible, the amount of labor required by Kronecker's method is altogether too great. As a polynomial is completely determined by a sufficiently extended table of values, these values should suffice to determine the reducibility or irreducibility of the polynomial. We can hardly expect to establish the reducibility of a polynomial of degree $n$, with fewer than $n+1$ entries in its table of values. For this reason criteria establishing the reducibility of a polynomial are unknown. No such criteria are established in the present paper. On the other hand, one entry in the table of values of a polynomial may be sufficient to establish its $i r$ reducibility. The present paper is concerned with criteria of this sort.

One such criterion is available: $\dagger$ if for a sufficiently large integer $h, f(h)$ is a prime, where $f(x)$ is a polynomial with inte-

* Presented to the Society, March 30, 1934.

† See P. Stäckel, Journal für Mathematik, vol. 148 (1918), p. 109; Pólya and Szegö, A ufgaben und Lehrsätze, vol. 2, p. 137, Ex. 127. 